\title{
A pilot study on the ontogeny of digestive physiology in Japanese macaques (Macaca fuscata)
}

\author{
Sawada, Akiko ; Sakaguchi, Ei ; Clauss, Marcus ; Hanya, Goro
}

\begin{abstract}
An assumption based on the Jarman-Bell principle suggests a positive relationship between body size and the digestive efficiency in animals, where smaller animals are less effective at digesting fibrous food due to shorter digesta passage. To examine the effect of body size within a species and explore a potential physiological background of ontogenetic diet shifts, we measured food intake, digestibility, digesta passage and gut fill in nine Japanese macaques, including three juveniles/subadult animals. Although these three showed a comparable digestive efficiency as the older animals on a low-fiber diet, they did not achieve the long retention times of adults in spite of similar levels of indigestible food intake and gut capacity. While the limited sample size would not allow generalized conclusions on ontogenetic digestive development in primates, this study suggests additional, yet unexplored effects other than food intake, digestion and gut capacity on digesta retention during ontogeny.
\end{abstract}

DOI: https://doi.org/10.1016/j.mambio.2012.06.002

Posted at the Zurich Open Repository and Archive, University of Zurich

ZORA URL: https://doi.org/10.5167/uzh-64981

Journal Article

Accepted Version

Originally published at:

Sawada, Akiko; Sakaguchi, Ei; Clauss, Marcus; Hanya, Goro (2012). A pilot study on the ontogeny of digestive physiology in Japanese macaques (Macaca fuscata). Mammalian Biology - Zeitschrift für Säugetierkunde, 77(6):455-458.

DOI: https://doi.org/10.1016/j.mambio.2012.06.002 
1 A pilot study on the ontogeny of digestive physiology in Japanese macaques

2 (Macaca fuscata)

3

4

5 AKIKO SAWADA $^{1}$, EI SAKAGUCHI ${ }^{2}$, MARCUS CLAUSS $^{3}$ AND GORO HANYA ${ }^{1}$

6

$7 \quad{ }^{1}$ Primate Research Institute, Kyoto University, Inuyama, Japan

$8{ }^{2}$ Graduate School of Natural Science and Technology, Okayama University, Okayama,

9 Japan

$10{ }^{3}$ Clinic for Zoo Animals, Exotic Pets and Wildlife, University of Zurich, Zurich,

11 Switzerland

12

$13{ }^{*}$ Correspondence to: Akiko SAWADA, Primate Research Institute, Kyoto University,

14 Kanrin 41-2, Inuyama, Aichi, 484-8506, Japan. Telephone: +81-568-63-0545,

15 facsimile: +81-568-63-0564, e-mail: a_sawada@pri.kyoto-u.ac.jp 


\section{ABSTRACT}

An assumption based on the Jarman-Bell principle suggests a positive relationship between body size and the digestive efficiency in animals, where smaller animals are less effective at digesting fibrous food due to shorter digesta passage. To examine the effect of body size within a species and explore a potential physiological background of ontogenetic diet shifts, we measured food intake, digestibility, digesta passage and gut fill in nine Japanese macaques, including three juveniles/subadult animals. Although these three showed a comparable digestive efficiency as the older animals on a low-fiber diet, they did not achieve the long retention times of adults in spite of similar levels of indigestible food intake and gut capacity. While the limited sample size would not allow generalized conclusions on ontogenetic digestive development in primates, this study suggests additional, yet unexplored effects other than food intake, digestion and gut capacity on digesta retention during ontogeny.

Key words: body size; digesta passage time; mean retention time; digestibility; gut capacity 


\section{INTRODUCTION}

The so-called Jarman-Bell principle suggests that smaller animals consume higher quality foods than larger animals, because they have higher relative energetic requirements (per unit body mass), and because larger animals with their higher absolute requirements have to rely on abundant (and hence lower quality) foods (Gaulin 1979). The principle has been invoked to explain inter-specific patterns of niche separation in primates, where small species tend to feed more on higher-quality but less abundant foods (insects) while larger species rely on lower-quality but more abundant foods (leaves) (Kay 1984; Sailer et al. 1985), or intra-specific patterns of niche separation in sexually dimorphic species (Barboza and Bowyer 2000) or between juveniles and adults (Agetsuma 2001; Hanya 2003).

The mechanical background of the Jarman-Bell principle has been sought in a systematic increase in digesta retention time with increasing body mass (Demment and Van Soest 1985), which would allow larger animals to digest high-fiber material more thoroughly, i.e. achieve higher digestive efficiencies. This positive relationship between body mass $(\mathrm{BM})$ and ingesta retention time was reported in both ruminants (from small antelopes to giraffes) and hindgut fermenters (from hares to elephants) (Illius and Gordon 1992). However, the validity of this relationship has been questioned on the basis of empirical data on primates (Lambert 1998; Clauss et al. 2008) and herbivorous mammals in general, with the possible exception of caecum fermenters (Clauss et al. 2007; Müller et al. 2011; Steuer et al. 2011). Empirical data also does not suggest an increase in digestive efficiency with BM in small rodents (Justice and Smith 1992), ruminants (Pérez-Barberìa et al. 2004), or large herbivores in general (Clauss et al. 2009). Digestive disadvantages of increasing BM have been demonstrated such as an 
increase in digesta particle size (Fritz et al. 2009) and methane losses per unit food or energy intake (Franz et al. 2011). What such large-scale comparisons do not evaluate is whether size-driven differences in digestive physiology occur during ontogeny within a species.

In free-ranging primates, different observations have been made regarding an influence of BM and diet selection. Juvenile Japanese macaques, for example, consumed animal matter more often and would not eat the high amounts of fibrous foods adult males consume (Agetsuma 2001; Hanya 2003). Female squirrel monkeys ate arthropods significantly more often than males during most of year (Boinski 1988). Such intra-specific differences, however, were not demonstrated in gorilla (Rothman et al. 2008) and orangutan (Van Schaik 1999). Nevertheless, even if differences in diet quality between juvenile and adult animals therefore cannot be linked to a principle that dominates all species, the question remains whether such differences - when observed can be linked to ontogenetic changes in the digestive physiology of the species in question.

In the course of a study on the digestive physiology of Japanese macaques (Macaca fuscata) (Sawada et al. 2011), we examined dry matter intake (DMI), digesta passage time (expressed as mean retention time; MRT), apparent dry matter digestibility $(\mathrm{aD} D M)$ and dry matter gut contents (DMC) in adult male Japanese macaques $(\mathrm{n}=4)$ on two diets (high and low fibre, (neutral detergent fibre NDF $37.5 \%$ and $13.6 \%$ in dry matter, respectively) and two intake levels (ad libitum and restricted). Originally, we had planned to have more animals, including juveniles and females, to cover a wider range of body sizes. However, in parallel to the observed higher feeding selectivity in the wild, the younger animals refused the high-fiber food, could only be assessed when 
feeding ad libitum on low-fiber pellets, and were therefore excluded from the final data evaluation that focused on four adult males. Nevertheless, the additional measurements in five animals, including two juvenile and one subadult, on the low-fibre ad libitum diet provide some tentative insight into a potential physiological background of ontogenetic diet shifts in macaques.

\section{METHODS}

All experimental procedures, including adaptation periods, the measurement of food intake, faecal collection regime, and calculation of apparent digestibility, mean retention time of solute and particle markers, and dry matter gut fill, followed the methods described in the previous study (Sawada et al. 2011). Pellets were offered as $250 \mathrm{~g}$ as fed per day, and were never consumed completely. Nutrient composition of the pellets comprised, on an as fed basis, $25.9 \%$ crude protein, $4.7 \%$ crude fat, $13.6 \%$ neutral detergent fiber and $5.7 \%$ crude ash. Water was available ad libitum. This study adhered to the KUPRI "Guide for the Care and Use of Laboratory Primates", and was approved by the ethics committee of KUPRI.

Due to the relatively low age and much smaller body size of juveniles $(\mathrm{N}=2)$ and subadult ( $\mathrm{N}=1)$ compared to others (Table 1), we combined these three animals as one age class (juvenile/subadult). Correlations between digestive parameters and body mass were tested by Pearson's correlation coefficient, limiting all available data from the previous and this study to the low-fiber diet on the ad libitum intake level only, to assure comparability between the data gained during the previous study and this one. All correlations were performed including and excluding the juvenile/subadult group. Analyses were performed in PASW 18.0 (SPSS Inc., Chicago, IL); the significance 
level was set to 0.05 , considering values between 0.05 and 0.08 as trends.

107

108

109

110

111

112

113

114

115

116

117

118

119

120

121

122

123

124

125

126

127

128

129

\section{RESULTS}

The three juvenile/subadult animals had a higher relative DMI, similar aD DM and relative DMC, and shorter particle and solute MRT than adult animals (Table 1).

Absolute DMI increased with BM among all animals $(\mathrm{R}=0.930, \mathrm{p}<0.001)$ and adults only $(\mathrm{R}=0.792, \mathrm{p}=0.060)$ for the ad libitum low fibre diet. There was no significant correlation between BM and aD DM (Fig. 1a). MRT increased with BM only in the dataset including the juvenile/subadult animals, but not among adult animals only (Fig. 1b). Absolute DMC increased with $B M$ among all animals $(R=0.921, p<0.001)$ but not among adults only $(\mathrm{R}=0.614, \mathrm{p}=0.194)$; relative $\mathrm{DMC}$ did not change significantly with BM in either group (Fig. 1c). The two additional adult animals of this study matched the pattern of indigestible intake and MRT reported in the previous study for adult macaques (Fig. 1d). The juveniles/subadult, w did not achieve the basal MRT that the adult animals displayed (Fig. 1d).

\section{DISCUSSION}

Due to the low number of young animals sampled, interpretations based on our data must be considered hypothetical rather than conclusive. The data could suggest a change in digestive physiology during ontogeny and hence body size increase, towards longer digesta retention times at similar levels of relative gut capacity and indigestible food intake. In the previous study, we had found that the intake of indigestible material is negatively related to MRT: the more indigestible material is ingested, the shorter the MRT. However, from a certain level of indigestible intake on, MRT did not drop any 
further below approximately 30 hours. Our data indicates that young animals do not follow this pattern precisely but have shorter retention times. Because digesta retention is, amongst other factors, a function of gut capacity (Hume 2005), we had first expected the difference between the juvenile/subadult and adults to be linked to a difference in this parameter. If the juvenile/subadult would have displayed a lower gut fill, one could have speculated that ontogenetic changes in gut capacity explain their shorter retention times. However, our data suggest that this was not the case here. Because the relative gut content mass did not differ between the juveniles/subadult group and the adults, other reasons than sheer gut capacity must be responsible for the shorter retention times in the younger animals. Our study therefore raises the interesting hypothesis that there might be an ontogenetic physiological constraint that prevents such long retention times in spite of an adequate gut capacity. Because a relationship between body mass and ingesta retention was not evident among adult animals, this hypothesized ontogenetic shift in ingesta retention could be considered of a principle or qualitative nature rather than just a continuous change that mirrors changes in body mass. This interpretation is in accord with more recent results that show that body mass is not the main predictor of digesta retention time across mammal species (Müller et al. 2011; Steuer et al. 2011). An attractive hypothesis that could explain the results of our study is a difference in gut peristalsis, possibly linked to an ontogenetic change in the gut microflora, including the methanogens. Franz et al. (2011) proposed that methane production might be one of the prerequisites of the long retention times usually associated with herbivory (or the consumption of low-quality food) based on the effects of methane on intestinal contractile activity in guinea pig and dog models (Pimentel et al. 2006). Thus, the presence of methanogens or the production of methane could 
theoretically be another factor required to achieve long digesta retention times, and the acquisition of an active methanogen microflora could be one of the reasons contributing to the shorter retention times in juveniles/subadults as compared to adults in this study.

This hypothesis could be tested experimentally by identifying (and quantifying) the components of the fecal microbiota, or by respirometry. In rabbits (Piattoni et al. 1996), chicken (Marounek and Rada 1998) and ruminants (Swainson et al. 2007; Staerfl et al. 2012), there are indications that young individuals have a less active methanogene microflora, the qcquisition of which may occur over a longer time period during ontogeny than the establishment of a fiber-digesting microflora in general. Evidently, other reasons for a difference in gut peristalsis between juveniles and adults should also be considered.

To conclude, while the results do not allow generalizations about ontogenetic digestive development in herbivores or primates in general, they may serve as a starting point to explore additional factors other than the interplay of intake, digestion and gut capacity on digesta retention. In particular, how and why juvenile animals are limited with respect to diets of low nutritional quality (Munn and Dawson 2006), may provide further insight into general digestive physiological principles.

\section{ACKNOWLEDGEMENTS}

This study complied with "Guide for the Care and Use of Laboratory Primates" by the KUPRI. The experimental protocol was approved by the Animal Welfare and Animal Care Committee of the KUPRI and Animal Research Committee of Kyoto University. All procedures adhered to the Japanese "Act on Welfare and Management of Animals". The authors are grateful to Yoshiaki Kamanaka, Mayumi Morimoto, 
Kiyonori Kumazaki and Naoko Hashimoto for their great support and help in carrying

out the experiments. We thank the members of the Laboratory of Animal Nutrition,

Okayama University, for help in marker analysis. We also appreciate the staff of

KUPRI giving us helpful comments and advice on this study. This study was supported

by grants from the Japan Ministry of Education, Culture, Sports, Science and

Technology (MEXT): Grants-in-Aid for JSPS Fellows, Grants-in-Aid for the Global

COE Program and AS-HOPE.

\section{REFERENCES}

Agetsuma N (2001) Relation between age-sex classes and dietary selection of wild Japanese monkeys. Ecological Research 16:759-763

Barboza PS, Bowyer RT (2000) Sexual segregation in dimorphic deer: a new gastrocentric hypothesis. Journal of Mammalogy 81:473-489

Boinski S (1988) Sex differences in the foraging behavior of squirrel monkeys in a seasonal habitat. Behavioral Ecology and Sociobiology 23:177-186

Clauss M, Nunn C, Fritz J, Hummel J (2009) Evidence for a tradeoff between retention time and chewing efficiency in large mammalian herbivores. Comparative Biochemistry and Physiology A 154:376-382

Clauss M, Schwarm A, Ortmann S, Streich WJ, Hummel J (2007) A case of non-scaling in mammalian physiology? Body size, digestive capacity, food intake, and ingesta passage in mammalian herbivores. Comparative Biochemistry and Physiology A 148:249-265

Clauss M et al. (2008) The influence of natural diet composition, food intake level, and body size on ingesta passage in primates. Comparative Biochemistry and Physiology A 150:274-281

Demment MW, Van Soest PJ (1985) A nutritional explanation for body size patterns of ruminant and nonruminant herbivores. American Naturalist 125:641-672

Franz R et al. (2011) Methane output of tortoises: its contribution to energy loss related to herbivore body mass. PLoS One 6:e17628

Fritz J, Hummel J, Kienzle E, Arnold C, Nunn C, Clauss M (2009) Comparative chewing efficiency in mammalian herbivores. Oikos 118:1623-1632

Gaulin SJC (1979) A Jarman-Bell model of primate feeding niches. Human Ecology 7:1-20

Hanya G (2003) Age differences in food intake and dietary selection of wild male Japanese macaques. Primates 44:333-339

Hume ID (2005) Concepts of digestive efficiency. In: Starck JM, Wang T (eds) Physiological and ecological adaptations to feeding in vertebrates. Science Publishers, Enfield NH, pp 43-58

Illius AW, Gordon IJ (1992) Modelling the nutritional ecology of ungulate herbivores: evolution of body size and competitive interactions. Oecologia 89:428-434

Justice KE, Smith FA (1992) A model of dietary fiber utilization by small mammalian herbivores, with empirical results for Neotoma. American Naturalist 139:398-416

Kay RF (1984) On the use of anatomical features to infer foraging behhavior in extinct primates. In: Rodman P, Cant J (eds) Adaptations for foraging in nonhuman primates. Columbio University Press, New York, pp 21-53

Lambert JE (1998) Primate digestion: interactions among anatomy, physiology, and feeding ecology. Evolutionary Anthropology 7:8-20

Marounek M, Rada V (1998) Age effect in in vitro fermentation pattern and methane production in the 
caeca of chickens. Physiological Research 47:259-263

Müller DWH et al. (2011) Phylogenetic constraints on digesta separation: variation in fluid throughput in the digestive tract in mammalian herbivores. Comparative Biochemistry and Physiology A 160:207-220

Munn AJ, Dawson TJ (2006) Forage fibre digestion, rates of feed passage and gut fill in juvenile and adult red kangaroos (Macropus rufus): why body size matters. Journal of Experimental Biology 209:1535-1547

Pérez-Barberìa FJ, Elston DA, Gordon IJ, Illius AW (2004) The evolution of phylogenetic differences in the efficiency of digestion in ruminants. Proceedings of the Royal Society B 271:1081-1090

Piattoni F, Demeyer DI, Maertens L (1996) In vitro study of the age-dependent caecal fermentation pattern and methanogenesis in young rabbits. Reproduction Nutrition Development $36: 253-261$

Pimentel M et al. (2006) Methane, a gas produced by enteric bacteria, slows intestinal transit and augments small intestinal contractile activity. American Journal of Physiology 290:G1089-G1095

Rothman J, Dierenfeld E, Hintz H, Pell A (2008) Nutritional quality of gorilla diets: consequences of age, sex, and season. Oecologia 155:111-122

Sailer L, Gaulin S, Boster J, Kurland J (1985) Measuring the relationship between dietary quality and body size in primates. Primates 26:14-27

Sawada A, Sakaguchi E, Hanya G (2011) Digesta passage time, digestibility, and total gut fill in captive Japanese macaques (Macaca fuscata): effects food type and food intake level. International Journal of Primatology 32:390-405

Staerfl SM, Zeitz JO, Kreuzer M, Soliva CR (2012) Methane conversion rate of bulls fattened on grass or maize silage as compared with the IPCC default values, and the long-term methane mitigation efficiency of adding acacia tannin, garlic, maca and lupine. Agriculture, Ecosystems and Environment 148:111-120

Steuer P et al. (2011) Is there an influence of body mass on digesta mean retention time in herbivores? A comparative study on ungulates. Comparative Biochemistry and Physiology A $160: 355-364$

Swainson NM, Hoskin SO, Clark H, Lopez-Villalobos N (2007) The effect of age on methane emissions from young, weaned red deer (Cervus elaphus) stags grazing perennial-ryegrass (Lolium perenne)-based pasture. New Zealand Journal of Agricultural Research 50:407-416

Van Schaik C (1999) The socioecology of fission-fusion sociality in Orangutans. Primates 40:69-86 
260 Table 1. Sex, body mass (BM), age, dry matter intake (DMI), mean retention time 261 (MRT) of particle (Cr) and solute (Co) markers, apparent digestibility of dry matter (aD 262 DM) and relative dry matter gut fill (rDMC, in \% of BM) on an ad libitum, low-fiber $263 \operatorname{diet}(13.6 \%$ NDF in DM) in Japanese macaques (Macaca fuscata).

\begin{tabular}{|l|c|c|c|c|cc|cc|c|c|}
\hline \multirow{2}{*}{ Animal } & Sex & BM & Age & Age class & \multicolumn{2}{|c|}{ DMI } & \multicolumn{2}{|c|}{ MRT (h) } & aD DM & rDMC \\
\cline { 2 - 10 } & & $\mathbf{( k g})$ & $\mathbf{( y e a r s )}$ & & $(\mathrm{g} / \mathrm{d})$ & $\left(\mathrm{g} \mathrm{kg}^{-0.75} \mathrm{~d}^{-1}\right)$ & $\mathrm{Cr}$ & Co & $(\%)$ & $(\% \mathrm{BM})$ \\
2 & male & 2.6 & 1.0 & Juvenile & 117.9 & 57.6 & 26.1 & 26.5 & 89 & 2.72 \\
3 & female & 2.7 & 1.0 & Juvenile & 93.2 & 44.2 & 22.5 & 27.5 & 86 & 1.84 \\
4 & male & 5.9 & 4.1 & Subadult & 180.8 & 47.8 & 23.5 & 26.0 & 84 & 1.74 \\
5 & male & 8.8 & 6.1 & Adult & 192.2 & 37.6 & 34.4 & 37.1 & 85 & 1.80 \\
\hline $6-9 *$ & female & 9.6 & 8.1 & Adult & 184.7 & 33.9 & 53.7 & 27.0 & 84 & 2.50 \\
$($ mean \pm SD $)$ & male & 13.7 & 10.8 & Adult & 235.1 & 33.7 & 44.1 & 41.2 & 83 & 1.87 \\
& & \pm 1.9 & \pm 1.9 & & \pm 0.2 & \pm 4.0 & \pm 6.9 & \pm 8.0 & \pm 3 & \pm 0.29 \\
\hline
\end{tabular}


a

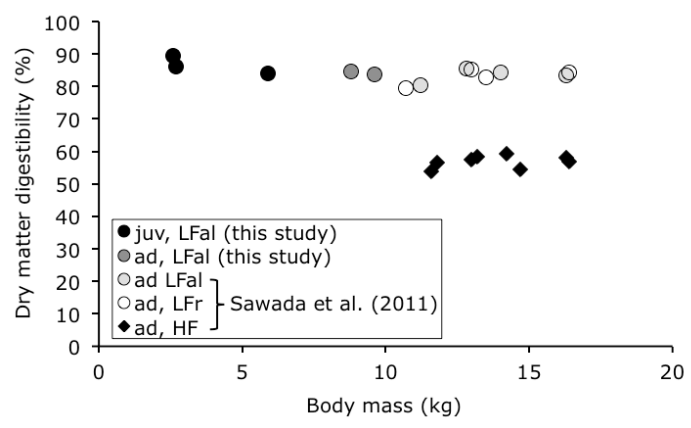

C

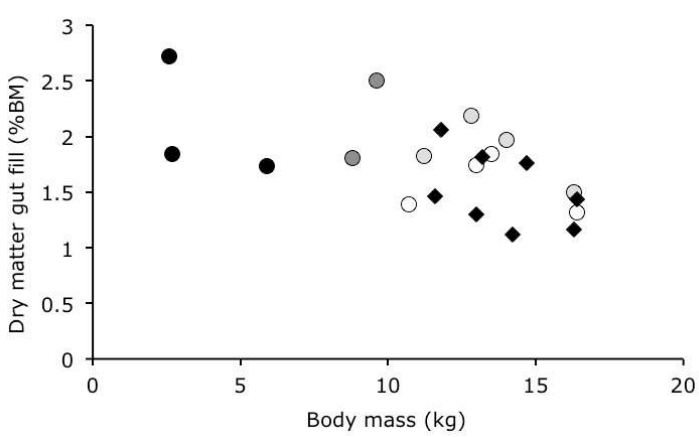

b
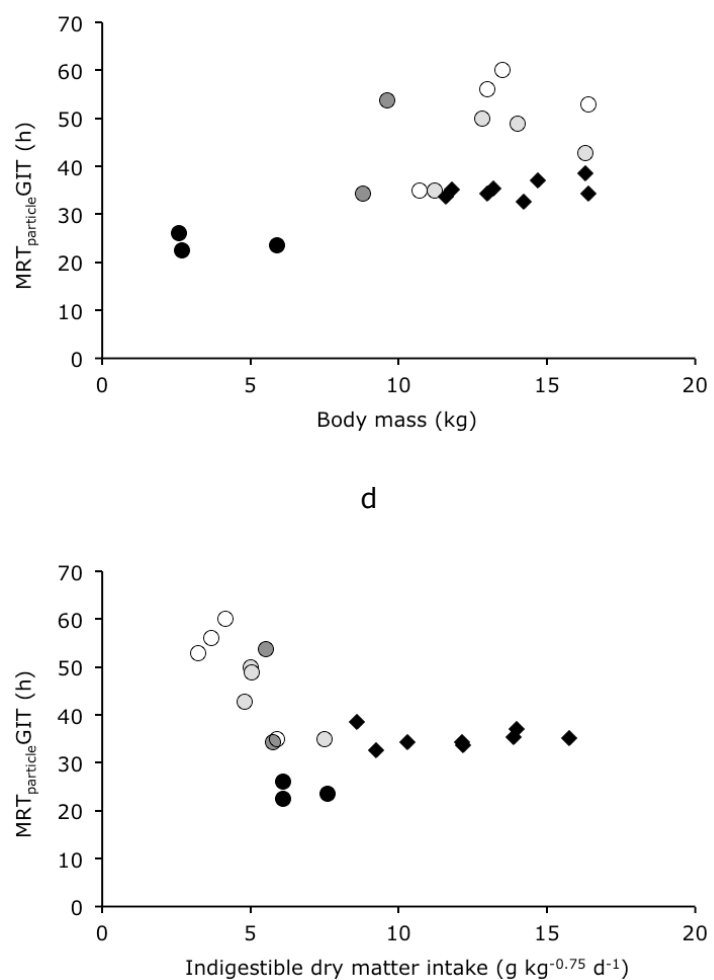

Figure 1. Results of juveniles and subadult (black dots) and adult animals (dark grey symbols) from this study as compared to those of adult animals on the same diet and intake level (low fibre, ad libitum intake LFal; light grey symbols) and in three other experiments (low fibre restricted intake, LFr, open symbols, and high fibre at two intake levels, black diamonds) from Sawada et al. (2011). a) Relationship between body mass (BM) and achieved dry matter digestibility; note that smaller animals refused to ingest the high fiber (i.e. less digestible) diet. For LFal, the relationship of BM and aD DM

274 was not significant for all animals $(\mathrm{R}=-0.590, \mathrm{p}=0.095)$ or for adult animals only $275(\mathrm{R}=0.042, \mathrm{p}=0.937)$. b) Relationship between $\mathrm{BM}$ and particle mean retention time

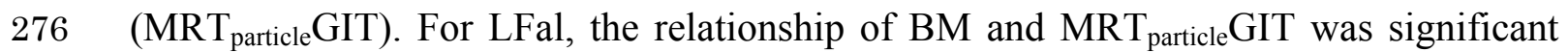
277 for all animals $(\mathrm{R}=0.744, \mathrm{p}=0.014)$ but not for adult animals only $(\mathrm{R}=0.205, \mathrm{p}=0.697)$; results were similar for $\mathrm{MRT}_{\text {particle }} \mathrm{GIT}$ for all animals $(\mathrm{R}=0.747, \mathrm{p}=0.021)$ and adult 

animals only ( $\mathrm{R}=0.540, \mathrm{p}=0.268$ ) (data not shown). c) Relationship between $\mathrm{BM}$ and 280 the relative dry matter content (DMC) of the gut. For LFal, the relationship of BM and $281 \mathrm{aD}$ DM was not significant for all animals $(\mathrm{R}=-0.397, \mathrm{p}=0.290)$ or for adult animals 282 only $(\mathrm{R}=-0.504, \mathrm{p}=0.308)$. d) Relationship between the indigestible dry matter intake 283 and particle mean retention time. Note a general biphasic pattern (already described in 284 Sawada et al. 2011) of a decrease in MRT with increasing indigestible intake, which 285 levels off at a certain point, and note that the juvenile/subadult animals do not seem to 286 follow this levelling-off of the adults. 